\title{
Contributions of the external field to the observatory annual means and a proposal for their corrections
}

\author{
Giuli Verbanac ${ }^{1,2}$, Hermann Lühr ${ }^{1}$, Martin Rother $^{1}$, Monika Korte $^{1}$, and Mioara Mandea ${ }^{1}$ \\ ${ }^{1}$ GeoForschungsZentrum Potsdam, Telegrafenberg, 14473 Potsdam, Germany \\ ${ }^{2}$ Faculty of Science, University of Zagreb, Horvatovac bb, 10000 Zagreb, Croatia
}

(Received July 31, 2006; Revised November 7, 2006; Accepted November 11, 2006; Online published May 7, 2007)

\begin{abstract}
In this study we separate, interpret and explain magnetospheric and ionospheric signals present in the observatory annual means. The data from 46 European geomagnetic observatories collected over 42 years (1960-2001) are used. To characterise the various field components, we use predictions from latest magnetic field models. The core field and its secular variation are described by the CM4 model, and the magnetospheric contributions are successfully removed by parameterising the POMME model with the Dst index. We regard the remaining signal as being caused by ionospheric currents. The annual averages of the Sq variation estimated by the CM4 model are subtracted from the residuals. A remaining variation in anti-phase with the magnetic activity index Ap finally can be removed with a function properly scaled by Ap and Dst. We offer an objective procedure to suppress the external field contributions in the annual means to an uncertainty level of $\pm 2 \mathrm{nT}$. Except for the ionospheric currents, this could be achieved by applying recent magnetic field models, which shows that the quality of present day models is sufficient to correct observatory data for average external field contributions. Understanding the signal contained in the annual means is a prerequisite for obtaining reliable and physically meaningful results when such data are used in studies of the core field and its secular variation.
\end{abstract}

Key words: Geomagnetic field, observatory annual means, external field, ring current, ionospheric currents, magnetic field models.

\section{Introduction}

Observatory annual means, defined as the average of all days of a year and all times of a day, are assumed to reflect the core field only and have been widely used in different studies of the internal geomagnetic field: modelling the core field (Barraclough et al., 1978), secular variation (SV) studies (Langel et al., 1986), regional modelling of SV (Korte and Holme, 2003) and induction studies (McLeod, 1992). It was recognized in the past (Yukutake and Cain, 1987) that observatory annual means are affected by the external field which varies with the solar cycle.

For accurate modelling of the main geomagnetic field it is essential to use a dataset which reflects as much as possible the core contribution. Nevertheless, annual means are sometimes used without prior correction for the external field. Moreover, contributions coming from outside the Earth have not been properly investigated to date.

Verbanac et al. (2006a) presented a study of the annual mean values of the $X$ (northward), $Y$ (eastward) and $Z$ (vertically downward) components from 46 European geomagnetic observatories over 42 years (1960-2001) that aimed at detecting long-term deviations in secular variation compared to current global models. They found that the external field descriptions included in the comprehensive CM4 model (Sabaka et al., 2004) could not sufficiently reduce

Copyright (c) The Society of Geomagnetism and Earth, Planetary and Space Sciences (SGEPSS); The Seismological Society of Japan; The Volcanological Society of Japan; The Geodetic Society of Japan; The Japanese Society for Planetary Sciences; TERRAPUB. these field contributions, which impeded the study of longterm internal field features. Consequently, they developed an empirical procedure for estimating the external field contributions in the data that is suitable for the region of interest.

Our aim here is to systematically study and explain the different geomagnetic field contributions from sources external to the Earth which are contained in the observatory annual means. We made use of the dense network of European geomagnetic observatories which, moreover, provide data over a time span long enough to allow for separation of the different influences of the external geomagnetic field. Only some of the observatories provide a continuous coverage of all epochs from 1960.5 to 2001.5. This time period is also covered by the CM4 model, which we are going to employ here. For this reason, the same data set as that used in the work of Verbanac et al. (2006a) is used again here. We are focusing on the northward, $X$, component, since this component is mostly influenced by the external field. In the previous paper an empirically determined function was used to correct for the external contributions. This is only applicable for regional studies. Here, we want to go a step further and provide a general description by investigating the sources and characteristics of various external field contributions by seeking for physical explanations of the remaining signals. To this end, the modules for external field contributions provided by the POtsdam Magnetic model of the Earth (POMME) (Maus et al., 2005) and CM4 were employed. We considered the annual means of differ- 
Table 1. Geomagnetic observatories considered in the present study. (The second numbers denote the representative observatory; see text).

\begin{tabular}{|c|c|c|c|}
\hline $\begin{array}{c}\text { Observatory } \\
\text { number }\end{array}$ & $\begin{array}{c}\text { Geomagnetic } \\
\text { longitude }\end{array}$ & $\begin{array}{c}\text { Coordinates } \\
\text { latitude }\end{array}$ & $\begin{array}{c}\text { IAGA } \\
\text { code }\end{array}$ \\
\hline 1 & 103.05 & 36.32 & PEG \\
\hline 2 & 108.86 & 38.28 & ISK \\
\hline 3 & 103.17 & 40.22 & ALM \\
\hline 4 & 107.01 & 40.57 & SFS \\
\hline 5 & 104.76 & 40.59 & PAG \\
\hline 6 & 107.43 & 42.29 & SUA \\
\hline $7 / 1$ & 94.37 & 42.53 & $\mathrm{AQU}$ \\
\hline 8 & 104.22 & 43.20 & SPT \\
\hline 9 & 102.29 & 43.27 & GCK \\
\hline 10 & 112.44 & 43.48 & ODE \\
\hline 11 & 98.85 & 43.51 & EBR \\
\hline 12 & 108.20 & 44.61 & COI \\
\hline 13 & 100.46 & 46.00 & THY \\
\hline $14 / 2$ & 94.14 & 46.39 & CTS \\
\hline 15 & 101.12 & 46.88 & HRB \\
\hline 16 & 99.64 & 46.93 & NCK \\
\hline 17 & 113.44 & 47.37 & KIV \\
\hline $18 / 3$ & 99.53 & 47.62 & WIK \\
\hline 19 & 107.15 & 47.78 & LVV \\
\hline $20 / 4$ & 94.68 & 48.49 & FUR \\
\hline $21 / 5$ & 97.70 & 48.83 & BDV \\
\hline $22 / 6$ & 94.22 & 50.10 & CLF \\
\hline $23 / 7$ & 105.31 & 50.17 & BEL \\
\hline 24 & 121.68 & 50.71 & MOS \\
\hline 25 & 112.99 & 51.42 & MNK \\
\hline 26 & 90.22 & 51.61 & MAB \\
\hline $27 / 8$ & 90.93 & 51.63 & DOU \\
\hline $28 / 9$ & 97.85 & 51.94 & NGK \\
\hline $29 / 10$ & 124.30 & 52.89 & BOX \\
\hline $30 / 11$ & 104.89 & 53.17 & HLP \\
\hline $31 / 12$ & 92.49 & 53.82 & WIT \\
\hline $32 / 13$ & 95.31 & 54.23 & WNG \\
\hline $33 / 14$ & 99.60 & 54.24 & HAD \\
\hline $34 / 15$ & 98.86 & 55.50 & BFE \\
\hline $35 / 16$ & 118.42 & 56.08 & LNN \\
\hline $36 / 17$ & 105.11 & 56.22 & VAL \\
\hline $37 / 18$ & 113.65 & 57.67 & NUR \\
\hline $38 / 19$ & 106.89 & 57.82 & LOV \\
\hline $39 / 20$ & 95.80 & 58.09 & ESK \\
\hline $40 / 21$ & 118.77 & 60.92 & OUL \\
\hline $41 / 22$ & 101.14 & 61.97 & DOB \\
\hline $42 / 23$ & 90.26 & 62.18 & LER \\
\hline 43 & 120.83 & 63.62 & SOD \\
\hline 44 & 116.37 & 65.06 & KIR \\
\hline 45 & 117.41 & 66.96 & TRO \\
\hline $46 / 24$ & 107.76 & 69.77 & LRV \\
\hline
\end{tabular}

ent indices, such as-Dst (storm time disturbance), F10.7 (solar flux influencing the conductivity of the ionosphere) and Ap (planetary geomagnetic activity)_and tested their relation to the residuals of the annual means. Our aim is to develop a method to correct the observatory annual means for external field variations. The core field, as predicted by CM4 model, was removed for convenience, solely to see these external signals more clearly. Finally, we suggest a procedure to remove the signals which do not belong to the internal field.

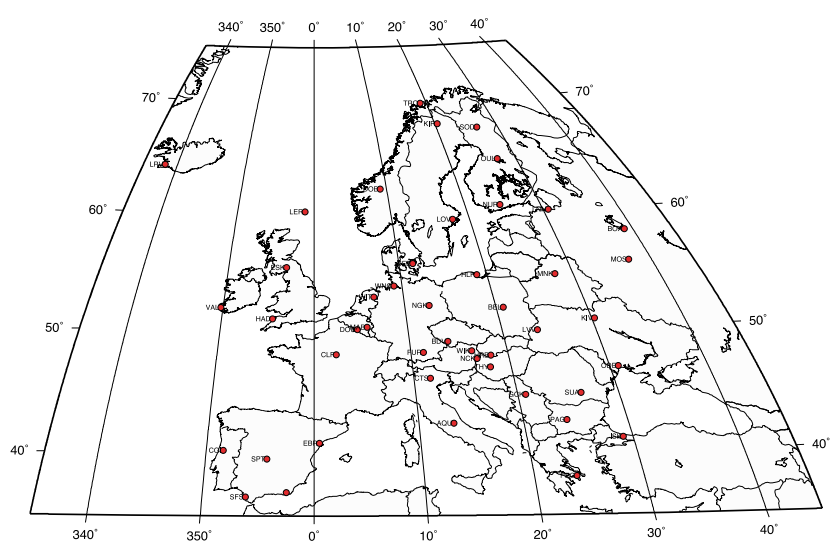

Fig. 1. Map of the geomagnetic observatories used in this study.

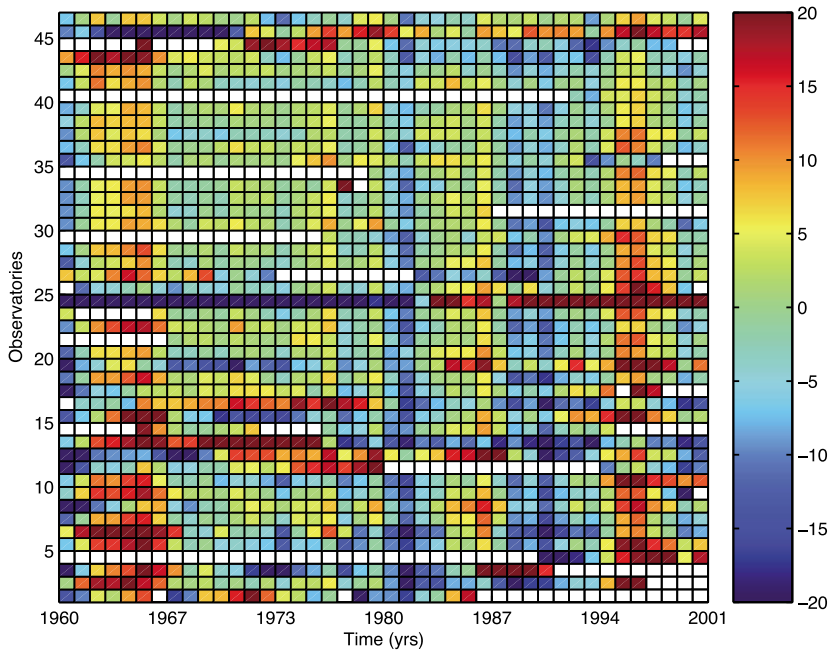

Fig. 2. Pictograms of $X$ component residuals after subtraction of the core field. The observatories (ordinate) are ordered by geomagnetic latitudes, with the number assignment as given in Table 1. Figure is taken from Verbanac et al. (2006a).

With this approach, our study shows a way to use corrected observatory annual means for different studies of the internal geomagnetic field.

\section{Data and Used Models}

Our study is based on observatory annual means and synthetic data obtained from the CM4 and POMME models for the European observatory locations. The time series over 42 years (1960-2001) is investigated. In this section we describe the various data sets.

\subsection{Observatory data}

The annual mean values of the $X$ component from 46 European geomagnetic observatories, which are given for epochs xxxx.5, i.e. the middle of the years, are used (http://www.geomag.bgs.ac.uk/gifs/annual_means.shtml).

Only 27 observatories reported values for all 42 years. For two locations, less than 20 years of data are available. A detailed description of the data set, including peculiarities in the data at some sites, are given in Verbanac et al. (2006a). 


\subsection{Data based on the CM4 model}

To estimate the core field at observatory locations, the CM4 model (Sabaka et al., 2004) is used. The model is valid for the period 1960-2002, covering the full time span of our interest. The CM4 model has been derived from quiet-time MAGSAT, POGO, Ørsted and CHAMP satellite data in combination with observatory hourly means. It is an attempt to co-estimate all the various field contributions at the same time-core field and lithospheric field as well as the external field generated by magnetospheric and ionospheric currents, together with the field-aligned currents, which couple the two external systems, and, finally, the currents induced in the conducting Earth. Moreover, the secular variation of the core field is described as a continuous function. This differs from the standard International Geomagnetic Reference Field (e.g. Macmillan et al., 2003), which provides updates only every 5 years. Using the CM4 code, we calculated the core field at each observatory location for all 42 epochs up to degree and order 14 and subtracted it from the annual means data to eliminate the core field and secular variation contribution. We also made use of the module within the CM4 which calculates the Sq currents and related induced magnetic fields.

\subsection{Data based on the POMME model}

To estimate the large-scale external fields at observatory locations we use the external field description included in the POMME version 2.5 (Maus et al., 2005). These contributions, including the ring, magneto-tail and magnetopause currents, can be estimated independently from the core field and thus can also be calculated for epochs outside the validity of the POMME-2.5 model, which is centered on 2002.0. A special feature of POMME-2.5 is that it treats the magnetospheric contributions in specific coordinate systems in which the currents are best organized (Maus and Lühr, 2005). This allows minimizing the number of parameters to describe the effects. The influence of the ring current, for example, is computed in Solar-Magnetic (SM) coordinates. For a separate treatment of the internal and external parts of the ring current effect, the Dst index is decomposed into the parameters Ist and Est (Maus and Weidelt, 2004). The Est, Ist indices have been kindly estimated for the whole time span since 1960 by S. Maus and are available at http://www.ngdc.noaa.gov/seg/geomag/est_ist.shtml. Note that at satellite altitude, the ring current index has to be scaled down by a factor of about 0.8 (Maus and Lühr, 2005, Table 2). To correct the observatory data, we made use of the full amplitude, as reflected by the Dst index. The contributions of the magneto-tail currents and of the solar wind are calculated in Geocentric-Solar-Magnetospheric (GSM) coordinates. The main part of this contribution averages out when annual means are considered. For the actual application in this study, we used POMME-2.5 to calculate hourly values for the external part at each observatory location in geographic coordinates. This external field module of POMME is time-independent, but parametrized by Est, Ist and IMF components. The hourly values are averaged over 1 year. As a result, we obtain the magnetospheric correction vectors for the annual means of all considered observatories.

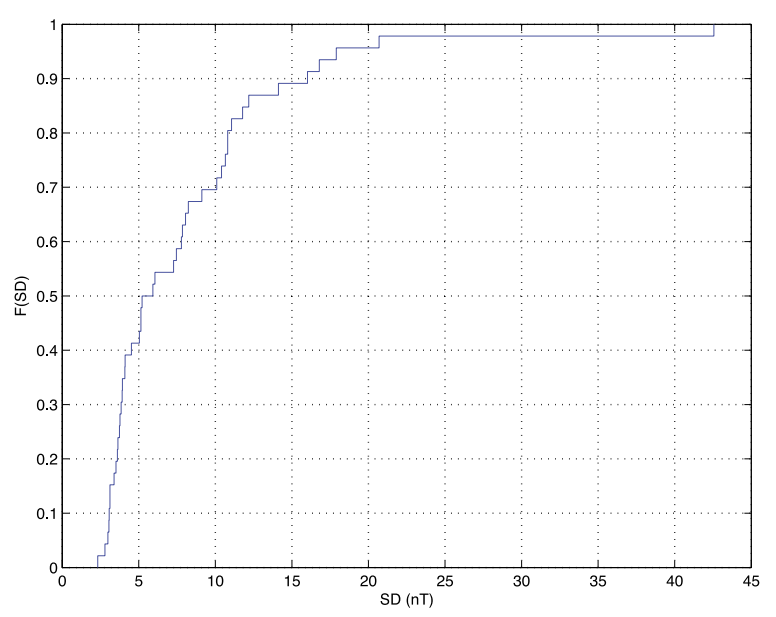

Fig. 3. Empirical cumulative distribution function (CDF) for the observatory annual means standard deviations, SD (see text for details).

\section{Method}

In this section we provide a description of the individual steps for removing the contributions from the different external field sources.

As noticed in Verbanac et al. (2006a), the residuals obtained by subtracting the predictions of the core field given by the CM4 model (http://dtam.gsfc.nasa.gov/cm/) from the observatory annual means reveal a similar short-term variation pattern at all observatories. This can be regarded as evidence for the external field influence present in the annual means (see Fig. 2). In the color-coded matrix, the observatories are ordered by geomagnetic latitude (ordinate), with the northern at the top and the southern at the bottom of the panel. Note that the white fields in the pictograms are years without reported measurements. For the assignment of observatories to the numbers, see Table 1 . The vertical stripes in the color-coded matrix, which are clearly recognizable, coincide with the times of solar minima and maxima. As a next step, we subtract the POMME magnetospheric field from the annual means. Remaining non-zero averages over the 42 years are eliminated from each time series in order to present all observatory values on the same color scale. The obtained distribution of residuals is shown in Fig. 4(a). Individual large values at some observatories are indicative of measurements errors. The vertical stripes of maxima and minima present in the data set of Verbanac et al. (2006a), linked to the solar cycle, do not show up clearly any longer, confirming that the solar cycle linked influence has been significantly reduced. There is one exception, however, around 1996, where the vertical stripe has become weaker but is still clearly visible. Furthermore, individual years 1977 and 1982 are somewhat outstanding.

For a careful investigation of the external contributions to the annual means, it is essential to identify and exclude those observatories which show large trends or obvious measurement errors. For that reason, we computed the standard deviations $(S D)$ of the residuals for each observatory using all the available years and obtained an empirical estimation of the cumulative distribution function (CDF) for the whole set of European observatories. The empirical CDF is defined as the probability that a considered variable has a 

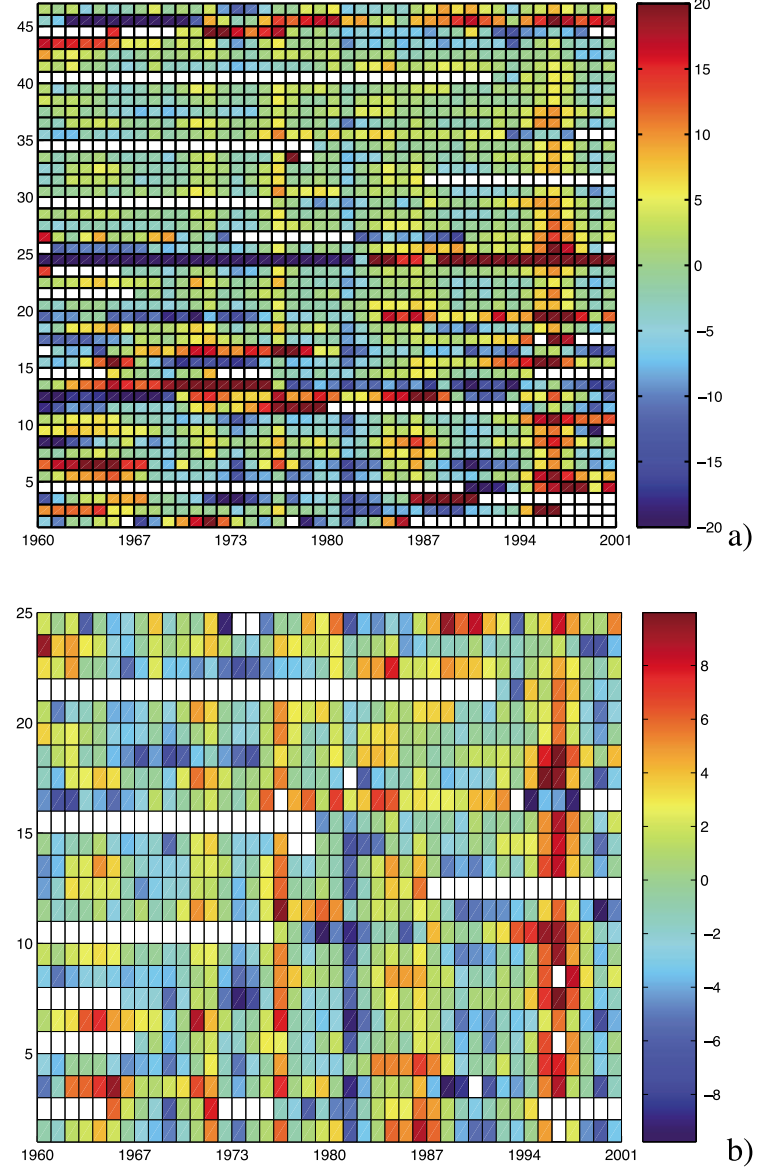

b)

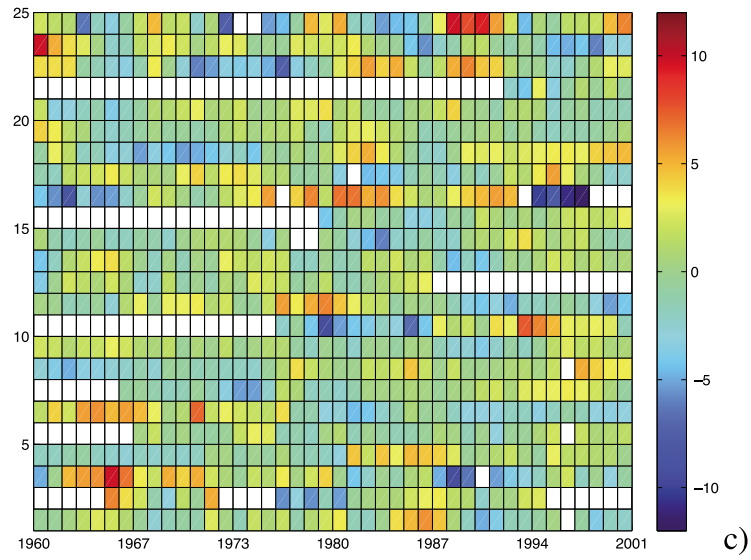

Fig. 4. Pictograms of the $X$ component residuals: (a) after subtracting the core field, as obtained by CM4 and the magnetospheric field, as predicted by the POMME model; (b) the same as in (a) but for representative observatories only (c) after subtracting the average variation (see text for details). The observatories are ordered by geomagnetic latitudes, with the relating number, as given in Table 1.

value less or equal to certain value $x$, and it is expressed in terms of the density function $F$ as:

$$
F(x ; \mu, \sigma)=\frac{1}{\sigma \sqrt{2 \pi}} \int_{-\infty}^{x} \exp \left(-\frac{(u-\mu)^{2}}{2 \sigma^{2}}\right) d u
$$

where $\mu$ is the mean and $\sigma$ is standard deviation of the considered variable which is, in our case, the observatory residuals $S D$, and the CDF gives the proportion of data points
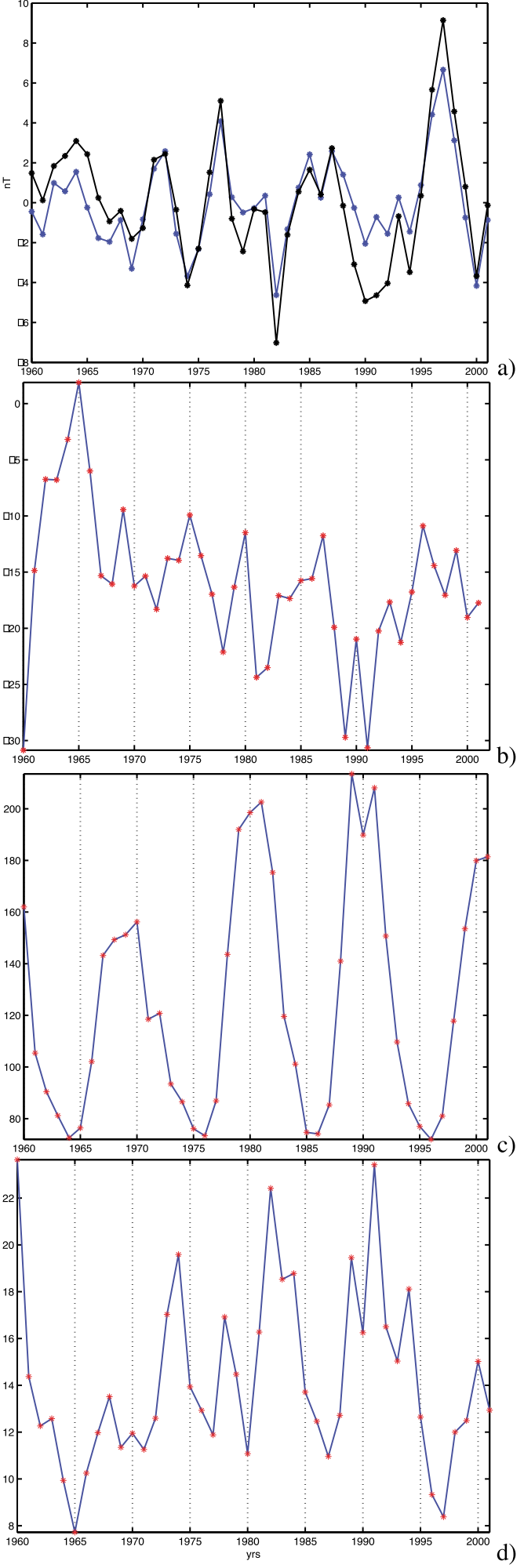

Fig. 5. (a) Blue: $X$ component residuals obtained by vertical averaging of all observatories, Res1 (see color-coded matrix in Fig 4(b)) and black: Res1 at NGK; annual average values of the indices: (b) Dst; (c) F10.7; (d) Ap.

less than each $S D$ value. From the CDF plot (Fig. 3), we found that at the level of about 0.5 the cumulative function is constant over a few $S D$ values. Observatories above this level were rejected, and the remaining $54 \%$ were considered in further analyses. This new set of observatories 
fortunately still covers the original range of geomagnetic latitudes. The color-coded matrix, with the reduced set of observatories, is shown in Fig. 4(b). In comparison with Fig. 4(a), the new plot looks much smoother, although the resolution has been enlarged by a factor of 2, supporting the criterion we have chosen for finding the representative set of observatories. From this set of observatories, a template for remaining common variations is constructed by vertical averaging over all locations for every year. The obtained average curve (hereafter named Res1) is shown as blue line in Fig. 5(a) and can be understood as the reflection of the persistent external field signal in the residuals. When subtracting the template from the field values in Fig. 4(b), we obtain an almost random distribution of the remaining values over the whole time-space domain, as seen in Fig. 4(c). This probably reflects mainly the actual measurements noise in the observatory annual means. From Fig. 4(c) we can conclude that the residual noise in the data of the selected representative observatory is quite low (few nT) and uncorrelated. For that reason, the averaged remaining variation (Res1), shown in Fig. 5(a) (blue curve), is considered as a real signal. In order to find the origin of these variations, panels (b), (c) and (d) of Fig. 5 show, for comparison, annual means of the indices Dst, F10.7 and Ap. The F10.7 index is a measure of solar radio flux and can be used to estimate solar extreme ultra-violet radiation, which controls ionospheric conductivity. The Ap index is a measure of the global geomagnetic activity per day. This linear measure is more suitable for our correlation study than the more commonly used quasi-logarithmic Kp index. There is no obvious correlation of the residuals with the annual averages of the Dst. The solar flux in Fig. 4(c) also exhibits quite a different signature. However, there is an apparent anti-correlation between the residuals and the Ap variations, which we suppose to be caused by ionospheric currents. It had been pointed out by Schmucker (1991) that the Sq currents make a non-vanishing contribution to the annual means. The amplitude of that value varies with the solar cycle. In order to also take this external contribution into consideration we make use of the module within the CM4 model, which calculates the effect of the Sq currents and the related induction effect. In Fig. 6 we plotted this synthetic Sq signal (red curve), computed for a central location (NGK), which is considered to be representative for the average value of the European network. When overplotting the Res1 at NGK (black curve) on top of the average Res1 in Fig. 5(a), one can see that both curves track each other quite well over the whole time-span, supporting the above mentioned assumption. The remaining signal (hereafter termed Res2) after removal of the Sq variation is shown in Fig. 6. This curve still shows significant amplitudes, which we believe are caused by further ionospheric currents. Although not mentioned by Schmucker (1991), we suggest an anti-correlation between the Ap index and the variations of Res 2 . It is probably not justified to expect a direct correlation between the residuals and the annual means of the Ap. The Ap is a global index of the geomagnetic activity and contains certain contributions of the ring current field, as described by Dst. In an earlier processing step, we have already corrected the observatory data for that

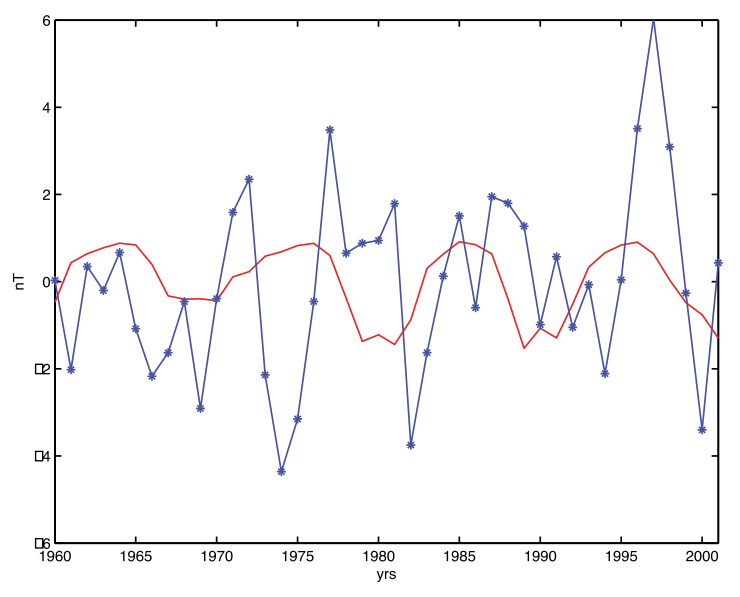

Fig. 6. The same residual signal as in Fig. 4(a) but corrected for the Sq effect (blue curve). The annual average of the Sq signal (red curve), as predicted by the CM4 model for the location of Niemegk.

magnetospheric effect. In order not to overcorrect the data, we performed a bi-variant correlation analysis taking into consideration the Ap index and regional ring current effect (Rc) simultaneously:

$$
\boldsymbol{\operatorname { R e s } 2}=a \cdot \mathbf{A p}+b \cdot \mathbf{R} \mathbf{c}+c+\text { error },
$$

where the coefficients $a, b$ and $c$ are determined by a least squares minimization of the error.

Here we use the ring current, Rc, part of the POMME magnetospheric description for NGK since it has a clear latitudinal dependence (Verbanac et al., 2006b). However, as Ap is a global index and the residual signal appears to be quite homogeneous for this considered region, the correlation is made for only one representative Rc location.

The analysis gave the following values: $a=-0.60$, $b=-0.49, c=3.84 \mathrm{nT}$ and a standard deviation of 1.54 $\mathrm{nT}$ for the error vector. The ionospheric signal of the north component, $X$, for the central part of Europe can therefore be expressed as:

$$
X_{\text {iono }}=\mathrm{Sq}-0.60 \cdot(\mathrm{Ap}+0.82 \cdot \mathrm{Rc})+3.84 \mathrm{nT} .
$$

In Eq. (3), the large contribution of the Rc variations to the Ap index becomes evident (Note that Rc has primary negative value). Furthermore, we see that there are additional ionospheric contributions to the annual means that are not covered by any of the present models, but which are reflected somehow by the activity index Ap. Just as a test we have also tried to co-estimate an additional scaling factor for the Sq signal in Eq. (3) (1.6 · Sq). This improved the standard deviation only slightly, by $2 \%$, but at the expense of an additional parameter. For that reason we did not pursue this approach any further. We subtract the above described Ap correction from the residuals Res2 (shown in Fig. 6) and obtain the final curve of residuals, which is shown in Fig. 7 as a blue curve and thereafter termed Res3. The signal varies predominantly within $\pm 2 \mathrm{nT}$. There are no features that are particularly outstanding. For comparison, Fig. 7 also contains average residuals of the representative observatories prior to the application any external field correction (green curve). A big improvement is already achieved by removing the ring current effect. This Res1, plotted as a dashed 


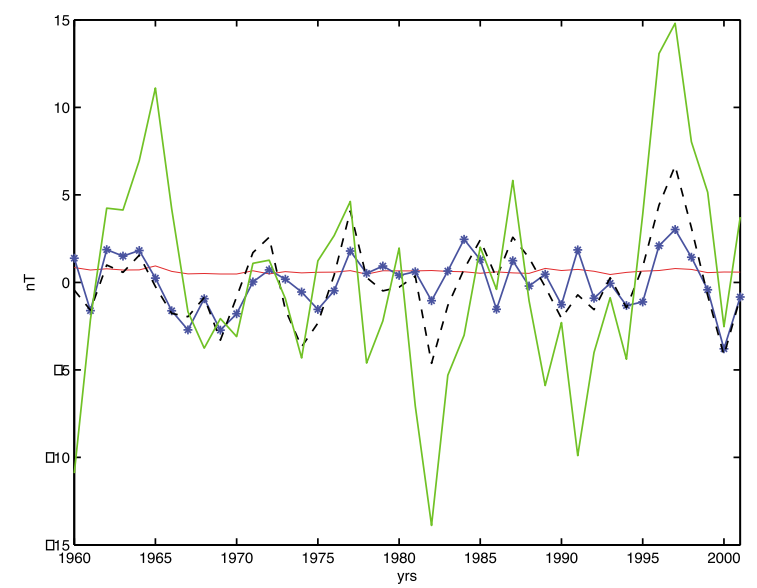

Fig. 7. Residual of the annual means $X$ component: prior to any external field correction (green curve); Res1 (black dashed curve); Res3 (blue curve). The red curve represents mean uncertainty of averaged annual means derived from the observatory residuals presented in Fig. 4(c).

black curve, exhibits a standard deviation of $2.3 \mathrm{nT}$, which is almost threefold less than that of the uncorrected green curve. Applying also the ionospheric correction gives the final residuals (Res3), which are drawn in blue. For Res3, we computed a standard deviation of $1.54 \mathrm{nT}$. The remaining red curve reflects the mean uncertainty of the averaged annual means used throughout this study. We computed it from the observatory residuals presented in Fig. 4(c) individually for each year. The obtained uncertainty level of 0.6 $\mathrm{nT}$ is fairly constant over the considered time span. Comparison of this number with the Res 3 curve (blue) suggests that even our final residuals contain some uncorrected external contributions.

\section{Interpretation and Discussion}

In the preceding sections we have described all of the considered field contributions and the models from which they were obtained. Here, we concentrate more on the physical separation of the residuals in the annual means. The core field with its secular variation is subtracted from the observatory data for convenience purposes only. The temporal changes of this component accumulated over 42 years are several fold larger than the influence of the external sources. A proper reduction clearly brings out the periodic character (11-year solar cycle) of the latter fields (cf. Fig. 2). Our primary aim was to identify the source currents responsible for these and to find a proper model for their reduction.

By applying the POMME-2.5 model, the magnetospheric field is expected to be strongly reduced, since we removed the Dst-related variations as a signature of the ring current behaviour as well as the magnetospheric tail fields. In addition to the ring current variation itself, its induction effects in the Earth's interior are taken into account by the POMME model. The stable contribution from the tail currents, 12.9 $\mathrm{nT}$, is aligned with $B_{z}$ in GSM coordinates, and the constant part of the ring plus magnetopause currents, $7.6 \mathrm{nT}$, is aligned with the dipole axis (Maus and Lühr, 2005). We are interested in the details of all external variations contained in the annual means. For that reason, we have aver- aged the residuals of representative European observatories after the POMME-2.5 model magnetospheric correction to obtain a reliable time series of the remaining variations in the data. The residual signal of the $X$ component, as shown in Fig. 5(a), contains a series of prominent peaks, but there is no more obvious correlation with the Dst annual averages (Fig. 5(b)). This indicates that the effect of magnetospheric currents has been removed reliably. We suppose ionospheric currents to be responsible for the remaining variations in the annual means (cf. Fig. 4(a)). An obvious candidate for such influence is of course the Sq current system. As was already pointed out by Schmucker (1991), the daily variation has a non-vanishing annual mean that varies with the solar cycle. The prediction of Sq annual means for central Europe derived from CM4 is shown in Fig. 6. Since this module of CM4 is parameterised by the solar flux index, F10.7, it is no surprise that the signal varies proportionally to that index. Our correlation analysis gives some indications that the solar cycle effect of Sq, as predicted by CM4, is somewhat underestimated. The significance of the result is, however, not sufficient to make suggestions for model improvements.

As pointed out in the previous section, even after the reduction of the $\mathrm{Sq}$ effect, there is an anti-correlation with the amplitude of the Ap index (cf. compare Figs. 5 and 6). This is an interesting relation, but also difficult to quantify. Ap is reflecting the range of variation in a 3-h interval; all non-harmonic disturbances are contributing to it (Siebert and Meyer, 1996). In particular, changes in the ring current intensity are also included. Therefore, Dst and Ap values cannot be considered to be fully independent. A clear anti-correlation is quite evident in Fig. 5 during the 1960s. This impression is well supported by our bi-variant correlation (Eq. (2)). It tells us that Ap has to be reduced by more than $80 \%$ of the Rc value at a mid-latitude station like NGK before a good correlation with the ionospheric residuals can be achieved. We do not regard this empirically derived relation between Ap and the magnetic field residuals as optimal. A dedicated parameter that reflects the ionospheric disturbances after reduction of the magnetospheric fields would be preferable. The POMME model has been shown to predict the magnetospheric field reasonably well. Another limitation of the Ap-related correction is that it is just a single number for the global activity, and it contains no information about the sign of the disturbance. The scaling factors for the correction of the annual means will therefore be latitude-dependent and for global application, it will be necessary to determine the relative importance of the different contributions for the different latitudes. The pending question is to what extend is Ap correlated to the auroral activity, the ring current intensity or the effects of the penetration electric field (Kikucki et al., 1996). The correction could then be performed for each source term independently rather than by comparison with a general activity index. However, such a study should preferably be carried out with data of higher temporal resolution, such as observatory hourly means, rather than with annual means where individual contributions probably average out to different degrees.

Despite these mentioned limitations for correcting the ef- 
fects of ionospheric currents, we can offer an objective procedure by which to suppress the external field contributions present in the annual means to a level of $\pm 2 \mathrm{nT}$ (cf. Fig. 7) for a continental-scale array. This can be regarded as sufficient since the uncertainty of representative European observatory annual means has been determined to range between 2 and 3 nT. Except for the ionospheric currents, the source terms could be derived from recent magnetic field models, which confirms their good quality with respect to external field contribution descriptions.

So far we have only considered the $X$ component, which is the one most severely affected by external sources. We regard this study as a test of concept and plan to further develop this technique for general use on a global scale. For improved understanding of the weaker external influences in the other components, however, it seems advisable to perform corrections on higher resolution data before averaging them to annual means.

\section{Conclusion}

In this study we have attempted to separate, interpret and explain all of the different external field signals present in observatory annual means.

We used data from 46 European geomagnetic observatories measured over 42 years (1960-2001). The dense distribution of geomagnetic observatories allows us to investigate a region from $35^{\circ}$ to $65^{\circ} \mathrm{N}$ in latitude and from $25^{\circ} \mathrm{W}$ to $40^{\circ} \mathrm{E}$ in longitude. The considered time span is long enough to cover a few solar cycles. To characterize various field components, we made use of predictions from the latest magnetic field models. The core field and its secular variation were described by the CM4 model. We successfully removed the magnetospheric contributions by the POMME2.5 model external field module parameterized by the Dst index. The remaining ionospheric signal obtained by averaging the data of representative observatories has a small amplitude $( \pm 6 \mathrm{nT})$ and varies approximately in anti-phase with the Ap index. For a further reduction of the remaining signal, we first estimated the annual averages of the Sq variation from the CM4 model and subtracted it from the residuals. We found that there was still a variation left which is in anti-phase with the Ap index. An empirically determined Ap-relation has finally been used to further reduce the residuals. Unfortunately, to date no suitable field model has been found to describe the responsible ionospheric currents. For the correction of this part we therefore cannot give a general recommendation. However, the remaining residuals of the European observatory annual means over the 42 years considered could be explained to a level of $\pm 2 \mathrm{nT}$.

The corrected data set of the annual means presents a good basis for core field studies. The present investigation shows that the external field signal contained in the observatory annual means is significant (see Fig. 7) and that their elimination is a prerequisite for obtaining reliable and physically meaningful results when such data are used in studies of the core field and its secular variation. An accurate estimation of the external field plays also a key role in identyfing spurious readings in observatory data.

This long time series of observatory annual means can furthermore be used to test the reliability of recent magnetic field models. The reduction of the magnetospheric fields with the POMME model seems to work to full satisfaction. The elimination of the Sq effect in the annual means based on the CM4 predictions is also within expectation, but may somewhat underestimate the amplitude. Not fully satisfying is the final correction based on the Ap index. Further investigation is required to develop suitable models that take the actual ionospheric source terms into consideration.

Acknowledgments. We thank S. Maus for calculating Est and Ist for the period under study. The European geomagnetic observatories are acknowledged for continuously providing their data. This study was performed during a research visit of M. Sc. G. Verbanac at GFZ Potsdam, which was supported by the Croatian Ministry of Science, Education and Sport as well as by the Deutsche Forschungsgemeinschaft (DFG).

\section{References}

Barraclough, D. R., J. M. Harwood, B. R. Leaton, and S. R. C. Malin, A definitive model of the geomagnetic field and its secular variation for 1965 , i. derivation of model and comparison with the IGRF, Geophys. J. R. Astr. Soc., 55, 111-121, 1978.

Kikucki, T., H. Lühr, T. Kitamura, O. Saka, and K. Schlegel, Direct penetration of the polar electric field to the equator during a Dp2 event, as detected by the auroral and equatorial magnetometer chains and the EISCAT radar, J. Geophys. Res., 101, 17161-17173, 1996.

Korte, M. and R. Holme, Regularization of spherical cap harmonics, Geophys. J. Int., 153, 253-262, 2003.

Langel, R. A., D. J. Nerridge, D. R. Barraclough, and S. R. C. Malin, Geomagnetic temporal change: 1903-1982. A spline representation, $J$. Geomag. Geoelectr., 38, 573-597, 1986.

Macmillan, S., S. Maus, T. Bondar, A. Chambodut, V. Golovkov, R. Holme, B. Langlais, V. Lesur, F. Lowes, H. Lühr, W. Mai, M. Mandea, N. Olsen, M. Rother, T. Sabaka, A. Thomson, and I. Wardinski, The 9th-generation International Geomagnetic Reference Field, Geophys. J. Int., 155, 1051-1056, 2003.

Maus, S. and H. Lühr, Signature of the quiet-time magnetospheric magnetic field and its electromagnetic induction in the rotating Earth, Geophys. J. International, 162(3), 755-763, 2005.

Maus, S., H. Lühr, G. Balasis, M. Rother, and M. Mandea, Introducing POMME, The Potsdam Magnetic Model of the Earth, in Earth Observation with CHAMP, Results from Three Years in Space, edited by C. Reigber, H. Lühr, P. Schwintzer, and J. Wickert, pp. 293-298, Springer, Berlin-Heidelberg, 2005.

Maus, S. and P. Weidelt, Separating the magnetospheric disturbance magnetic field into external and transient internal contributions using a 1D conductivity model of the Earth, Geophys. Res. Lett., 31, L12614, 2004.

McLeod, M., Signals and noise in magnetic observatory annual means: mantle conductivity and jerks, J. Geophys. Res., 97, 17261-17290, 1992.

Sabaka, T., N. Olsen, and M. Purucker, Extending comprehensive models of the Earth's magnetic field with Oersted and CHAMP data, Geophys. J. Int., 159, 521-547, 2004.

Schmucker, U., Solar cycle variations and corrected annual means for external effects at Fuerstenfeldbruck 1951-1968), Münchner Geophysikalische Mitteilungen, 5, 217-248, 1991.

Siebert, M. and J. Meyer, Geomagnetic activity indices, in The Upper Atmosphere, edited by W. Dieminger, G.K. Hartmann, and R. Leitinger, pp. 887-911, Springer-Verlag, Berlin-Heidelberg, 1996.

Verbanac, G., M. Korte, and M. Mandea, On long-term trends of the European geomagnetic observatory biases, Earth Planets Space, 2006a (submitted).

Verbanac, G., H. Lühr, and M. Rother, Evidence of the ring current effect in geomagnetic observatories annual means, Geofizika, 23, 13-20, 2006b.

Yukutake, T. and J. Cain, Solar cycle variations in the annual mean values of the geomagnetic components of observatory data, J. Geomag. Geoelectr., 39, 19-46, 1987.

G. Verbanac (e-mail: verbanac@irb.hr), H. Lühr, M. Rother, M. Korte, and M. Mandea 